\title{
ARTICLE \\ Clinical Study \\ Melanoma recurrence patterns and management after adjuvant targeted therapy: a multicentre analysis
}

\author{
Prachi Bhave (D) ${ }^{1}$, Lalit Pallan ${ }^{2}$, Georgina V. Long ${ }^{2,3}$, Alexander M. Menzies ${ }^{2,3}$, Victoria Atkinson ${ }^{4}$, Justine V. Cohen ${ }^{5}$, Ryan J. Sullivan ${ }^{5}$, \\ Vanna Chiarion-Sileni ${ }^{6}$, Marta Nyakas ${ }^{7}$, Katharina Kahler ${ }^{8}$, Axel Hauschild ${ }^{8}$, Ruth Plummer ${ }^{9}$, Claudia Trojaniello ${ }^{10}$, Paolo A. Ascierto ${ }^{10}$, \\ Lisa Zimmer $^{11}$, Dirk Schadendorf ${ }^{11}$, Clara Allayous ${ }^{12}$, Celeste Lebbe ${ }^{12}$, Andrea Maurichi ${ }^{13}$, Mario Santinami ${ }^{13}$, Severine Roy ${ }^{14}$, \\ Caroline Robert ${ }^{14}$, Thierry Lesimple ${ }^{15}$, Sapna Patel ${ }^{16}$, Judith M. Versluis ${ }^{17}$, Christian U. Blank ${ }^{17}$, Adnan Khattak ${ }^{18}$, \\ Andre Van der Westhuizen ${ }^{19}$, Matteo S. Carlino ${ }^{2,20}$, Mark Shackleton ${ }^{1,21}$ and Andrew Haydon (iD) ${ }^{1,21}$
}

BACKGROUND: Adjuvant targeted therapy (TT) improves relapse free survival in patients with resected BRAF mutant stage III melanoma. The outcomes and optimal management of patients who relapse after adjuvant $\Pi$ is unknown.

METHODS: Patients from twenty-one centres with recurrent melanoma after adjuvant $\Pi$ were included. Disease characteristics, adjuvant therapy, recurrence, treatment at relapse and outcomes were examined.

RESULTS: Eighty-five patients developed recurrent melanoma; nineteen (22\%) during adjuvant $\Pi$. Median time to first recurrence was 18 months and median follow-up from first recurrence was 31 months. Fifty-eight (68\%) patients received immunotherapy (IT) or $\Pi$ as 1 st line systemic therapy at either first or subsequent recurrence and had disease that was assessable for response. Response to anti-PD-1 ( \pm trial agent), combination ipilimumab-nivolumab, $\pi$ rechallenge and ipilimumab monotherapy was $63 \%$, $62 \% 25 \%$ and $10 \%$ respectively. Twenty-eight (33\%) patients had died at census, all from melanoma. Two-year OS was $84 \%$ for antiPD-1 therapy ( \pm trial agent), $92 \%$ for combination ipilimumab and nivolumab, $49 \%$ for $\Pi$ and $45 \%$ for ipilimumab monotherapy $(p=0.028)$.

CONCLUSIONS: Patients who relapse after adjuvant $\Pi$ respond well to subsequent anti-PD-1 based therapy and have outcomes similar to those seen when first line anti-PD-1 therapy is used in stage IV melanoma.

British Journal of Cancer (2021) 124:574-580; https://doi.org/10.1038/s41416-020-01121-y

\section{BACKGROUND}

The management of cutaneous melanoma has been revolutionised in the last decade. Targeted therapies (TT) inhibiting the MAPK pathway and immunotherapy (IT) with T-cell checkpoint inhibitors have each been demonstrated to prolong survival of patients with metastatic melanoma. ${ }^{1-5}$ As a result, these therapies are now mainstays of treatment for patients with unresectable stage III or stage IV disease.

Recent studies have tested adjuvant systemic $\Pi$ and IT for resected stage III/IV melanoma, with the aims of reducing melanoma recurrence and prolonging patient survival. ${ }^{6,7}$ Whilst $\Pi$ is reserved for patients with BRAF v600 mutant melanoma, IT may be used in patients irrespective of their BRAF status.
A number of randomised, Phase 3 trials have investigated IT as adjuvant treatment in melanoma. The EORTC-18071 trial compared adjuvant ipilimumab $10 \mathrm{mg} / \mathrm{kg}$ to placebo in patients with resected stage III disease and demonstrated an improvement in both relapse free survival (RFS, hazard ratio (HR) $0.76, p=0.0008$ ) and overall survival (OS) (HR $0.72, p=0.001)$. However, toxicity rates were high, with $45 \%$ of patients having grade $3-4$ adverse events (AEs), resulting in a third of patients discontinuing treatment. ${ }^{8-10}$ The Phase 3 E1609 trial examined the use of adjuvant ipilimumab at two doses $(3 \mathrm{mg} / \mathrm{kg}$ and $10 \mathrm{mg} / \mathrm{kg}$ ) and interferon alfa-2b. The lower ipilimumab dose improved OS compared to interferon, however, rates of grade 3-4 AEs were not negligible, and therefore single agent ipilimumab is rarely used in clinical practice. ${ }^{11}$

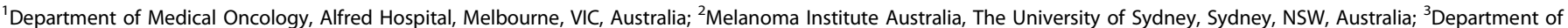

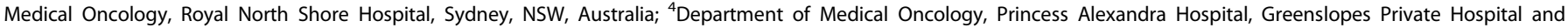

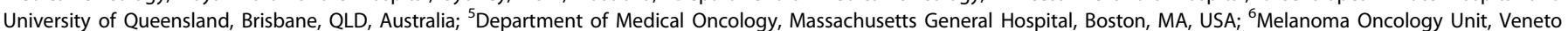

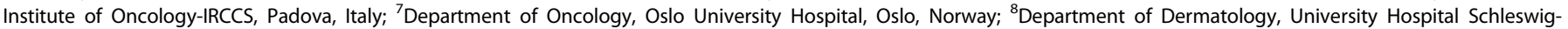

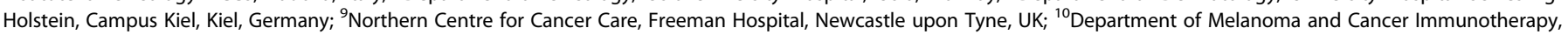

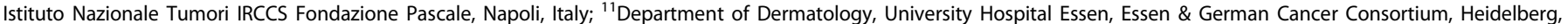

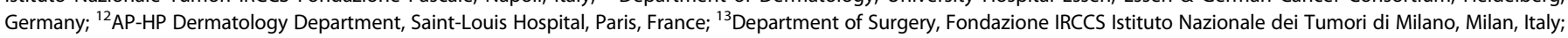

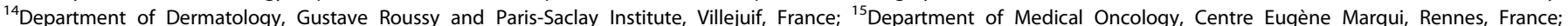

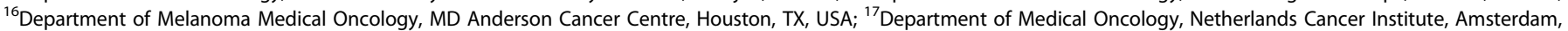

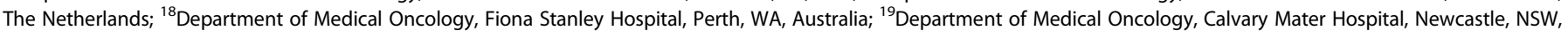
Australia; ${ }^{20}$ Department of Medical Oncology, Westmead Hospital, Sydney, NSW, Australia and ${ }^{21}$ Central Clinical School, Monash University, Melbourne, VIC, Australia Correspondence: Prachi Bhave (Prachi_Bhave@yahoo.com)
}

Received: 23 June 2020 Revised: 7 September 2020 Accepted: 2 October 2020

Published online: 22 October 2020 
The Checkmate-238 trial randomised patients with resected stage IIIB/C or IV disease to nivolumab $3 \mathrm{mg} / \mathrm{kg}$ or ipilimumab 10 $\mathrm{mg} / \mathrm{kg}$ for 1 year, finding an improvement in RFS (HR 0.66, $p<$ 0.0001 ) for nivolumab over ipilimumab, with only $14 \%$ of nivolumab patients having grade $3-4 \mathrm{AEs}{ }^{12,13}$ Discontinuation of nivolumab due to grade $3-4 \mathrm{AEs}$ occurred in $4.6 \%$ of patients, whereas $31 \%$ of ipilimumab patients discontinued treatment. Keynote-054 randomised patients with resected stage III melanoma to either pembrolizumab or placebo, also demonstrating an improvement in RFS with pembrolizumab (HR 0.57, $p<0.001$ ). Toxicity from pembrolizumab was comparable to that of nivolumab in Checkmate-238, with $14 \%$ of patients suffering a grade 3-4 adverse event. ${ }^{14}$ Of note, EORTC-18071 and Keynote054 excluded patients with $<1 \mathrm{~mm}$ of micro-metastatic disease in lymph nodes and all three adjuvant IT trials used the 7th edition of the AJCC melanoma staging system.

Two trials have examined adjuvant $T$ in BRAF mutant patients. The BRIM-8 trial included two cohorts of patients with resected melanoma (Cohort 1: stage IIC, IIIA, IIIB and Cohort 2: stage IIIC) that were randomised to single agent vemurafenib or placebo. $A$ two-cohort design was implemented to prevent the risk of stage IIIC patients driving the analysis due to their worse prognosis. The trial's primary end point of disease-free survival (DFS) underwent prespecified hierarchical testing, wherein DFS was tested in Cohort 2 before Cohort 1 . After one year of adjuvant vemurafenib, the trial did not meet statistical significance in Cohort 2, thereby rendering Cohort 1 's data also statistically insignificant. ${ }^{15}$ As a result, the use of adjuvant single agent BRAF inhibitors is not recommended.

The COMBI-AD trial tested dual agent dabrafenib and trametinib (DT) adjuvantly for one year in patients with resected stage III disease. The trial met its primary end point of RFS. At 4-years of follow-up, the RFS rate was $54 \%$ in the adjuvant DT arm, compared to $38 \%$ in the placebo arm, giving a HR of $0.49 .^{16,17}$ An interim analysis at three years demonstrated an OS of $86 \%$ with adjuvant DT compared to $77 \%$ for placebo (HR $0.57,95 \% \mathrm{Cl}$ $0.42-0.79, p=0.0006)$ although the $p$-value did not meet the prespecified significance boundary of 0.00019 . Final OS data is not yet available as the number of prespecified events has not yet been reached. Toxicity associated with adjuvant DT was not insignificant, with $31 \%$ of patients experiencing grade $3-4$ AEs due to DT, compared to $5 \%$ in the placebo group. Furthermore, $26 \%$ of patients prematurely ceased adjuvant DT due to toxicity; although, importantly, there were no treatment related deaths. As a result of this trial, dual agent $\Pi$ has become a standard treatment option for adjuvant therapy in patients with fully resected stage III BRAF mutant melanoma.

Although these trials have transformed treatment for stage III melanoma, the outcomes of patients who relapse after adjuvant therapy are not well described. For example, although current retrospective data suggest that response rates (RRs) to $\Pi$ are high in patients who relapse with unresectable disease after adjuvant $\mathrm{IT}^{18}$ little is known about the outcomes of patients who relapse after adjuvant $\Pi$, including their rates of response to subsequent lines of therapy and survival. We sought to address this in a multicentre, international cohort analysis.

\section{METHODS}

Institutional ethics board approval was obtained for the analysis Patients with resected stage III or IV melanoma (as per AJCC 8th edition) who recurred after receiving single or dual agent adjuvant $\Pi$ were included in this study.

Data was extracted from twenty-one melanoma centres worldwide. Patients were included by searching electronic and paper hospital records and institutional databases to identify patients enrolled in adjuvant $\Pi$ trials (BRIM-8 or COMBI-AD), those who accessed adjuvant $\Pi$ through patient access programs, as well as from hospital pharmacy records. If patients had participated in a randomised trial involving placebo, they were included if they had been unblinded at the time of melanoma recurrence and confirmed to have received adjuvant $\Pi$.

Patient records were retrospectively analysed and data on patient demographics were collected including age at diagnosis of resected stage III or IV melanoma and baseline melanoma characteristics such as tumour thickness, primary site, presence or absence of ulceration, mitotic rate and type of BRAF mutation. Information was recorded on diagnosis of stage III or IV melanoma using AJCC $8^{\text {th }}$ edition staging, on performance of completion lymph node dissection (CLND) and on the number of lymph nodes with metastatic disease. Details on adjuvant $\Pi$ were noted, including type used, duration and reason for cessation of therapy. Information was recorded on melanoma recurrence, including stage at recurrence, method of detection of recurrence, and sites of recurrent disease. Subsequent therapy was recorded, including modality of treatment, duration, response and complications. Imaging with $\mathrm{CT}$ of the chest, abdomen and pelvis or PET scan in combination with either a CT brain or MRI brain was performed. For patients on a clinical trial, imaging frequency occurred as per trial protocol; for patients not on trial, imaging occurred as per standard of care at each institution. Tumour response was evaluated by investigator review: any degree of tumour shrinkage was deemed a partial response, disease control was recorded as stable disease, resolution of all disease on CT or complete metabolic response on PET was considered a complete response and increase in tumour size or clinical deterioration due to melanoma was deemed progressive disease. Patients were followed until death or the data censorship date.

The Kaplan-Meier method was used to create survival curves. Comparisons between survival curves were made using the Log rank test. Cox regression was used to perform the multivariate analysis. All statistical analyses were performed using SPSS version 22.0.

\section{RESULTS}

Patient characteristics

Eighty-seven patients from centres across Australia, Europe and the United States of America met inclusion criteria. Two patients were excluded due to prolonged duration of adjuvant $\Pi$ (one for over 3 years and one for 1 year 8 months). Thus, the final analysis included 85 patients. Data was collected from January 2013 to September 2019.

Baseline patient characteristics are summarised in Table 1. The study population had a slight male predominance of $56 \%(n=48)$. Median age at time of diagnosis of resected stage III or IV melanoma was 47 years (range $22-74$ years). The majority $(n=81$, $95 \%$ ) of patients had primary cutaneous melanoma, with four (5\%) having no identifiable primary skin lesion (occult melanoma). $88 \%$ $(n=75)$ of patients had a BRAF V600E mutation whilst $7 \%(n=6)$ had a confirmed BRAF V600 mutation without subtyping. Of the included patients, $48 \%(n=41)$ were from Europe, with the remainder from Australia $(n=36,42 \%)$ or the United States of America $(n=8,9 \%)$. All patients were staged according to the $8^{\text {th }}$ edition of the AJCC staging system, with $61 \%$ of patients having stage IIIC disease and $2 \%$ having resected stage IV disease.

\section{Adjuvant therapy}

Details on adjuvant $\Pi$ received are summarised in Supplement 1. Eighty-one (95\%) patients had undergone complete lymph node dissection prior to commencement of adjuvant $\Pi$. Seventy-three (86\%) patients received adjuvant DT, one (1\%) received adjuvant vemurafenib and cobimetinib combination and eleven (13\%) received single agent vemurafenib. Four (5\%) patients received adjuvant radiotherapy (RT) before adjuvant $\Pi$, prior to first recurrence. Fifteen (18\%) patients received neoadjuvant followed 
Table 1. Baseline patient characteristics; melanoma staging as per AJCC 8th edition.

\begin{tabular}{lc}
\hline Characteristics & Patient number, \\
& $N=85(\%)$ \\
\hline Sex-no. (\%) & \\
Male & $48(56)$ \\
Female & $37(44)$ \\
Median age at diagnosis of resected stage III & $47(22-74)$ \\
or IV-year (range) & \\
Primary Site-no. (\%) & \\
Cutaneous & $81(95)$ \\
Occult & $4(5)$ \\
BRAF mutation-no. (\%) & \\
V600E & $75(88)$ \\
V600K & $3(4)$ \\
V600R & $1(1)$ \\
V600 (unspecified) & $6(7)$ \\
Patient origin-no. (\%) & \\
Australia & $36(42)$ \\
Europe & $41(48)$ \\
USA & $8(9)$ \\
Melanoma Stage at Adjuvant Treatment (AJCC 8 th edition)-no. (\%) & $83(98)$ \\
Stage III & $5(6)$ \\
IIIA & $21(25)$ \\
IIIB & $52(61)$ \\
IIIC & $5(6)$ \\
IIID & $2(2)$ \\
Resected stage IV & \\
\hline AJCC American Joint Committee on Cancer, USA United States of America. \\
${ }^{a}$ Six patients, all from a single centre in Europe, had a known BRAF V600 \\
mutation but subtype was unknown. \\
\end{tabular}

by adjuvant $\Pi$. Median duration of adjuvant therapy was 8.6 months. The majority (53\%) of patients ceased adjuvant $\Pi$ due to completion of the prescribed course.

Disease characteristics at first recurrence

Melanoma and patient characteristics at first recurrence after adjuvant $\Pi$ are summarised in Table 2 . Nineteen (22\%) patients recurred during adjuvant $\Pi$ and sixty-six (78\%) after cessation of adjuvant $\Pi$. Twenty-nine (34\%) patients recurred locoregionally, fifty $(59 \%)$ patients had distant recurrence and six $(7 \%)$ patients had both locoregional and distant recurrence.

The majority (88\%) of patients had an ECOG of 0 at recurrence and $57 \%$ of recurrences were detected on imaging, in the absence of symptomatic or clinically apparent disease. Median time to first recurrence was 17.7 months (1.7-53.6) and median time to development of distant recurrence was 19.2 months (3.1-56.4).

Treatment at first recurrence

At first recurrence, four (5\%) patients received RT only, all with palliative intent. Twenty-one (25\%) patients underwent surgery alone, of which $17(17 / 21,81 \%)$ had further recurrence of disease. Five $(6 \%)$ patients underwent surgery and RT combined, and all of these recurred subsequently. Thus, $85 \%(22 / 26)$ of patients who underwent surgery without systemic therapy at first recurrence developed a second recurrence of melanoma. Of the four patients who did not recur after surgery for first recurrence, three had distant disease and one had local disease at first recurrence.
Table 2. Disease characteristics at first recurrence including stage and method of detection of recurrence.

\begin{tabular}{lc}
\hline 1st Recurrence characteristics & $\begin{array}{l}\text { Patient number, } \\
N=85(\%)\end{array}$ \\
\hline $\begin{array}{l}\text { Median time to 1st recurrence- months } \\
\text { (range) }\end{array}$ & $17.7(1.7-53.6)$ \\
ECOG at recurrence-no. (\%) & \\
0 & $75(88)$ \\
1 & $8(9)$ \\
2 & $0(0)$ \\
3 & $1(1)$ \\
Unknown & $1(1)$ \\
Melanoma stage at 1st Recurrence (AJCC 8th edition)-no. (\%) \\
III & $26(31)$ \\
IIIB & $3(4)$ \\
IIIC & $18(21)$ \\
IIID & $5(6)$ \\
IV & $53(62)$ \\
M1a & $14(17)$ \\
M1b & $12(14)$ \\
M1C & $13(15)$ \\
M1d & $14(17)$ \\
Locoregional and distant recurrence & $6(7)$ \\
Recurrence primarily detected by-no. (\%) & \\
Symptoms & $10(12)$ \\
Clinical examination & $26(31)$ \\
Imaging & $49(57)$ \\
\hline
\end{tabular}

Twenty-eight (33\%) patients received systemic therapy alone at first recurrence, of which nineteen $(19 / 28,68 \%)$ developed a 2 nd recurrence. Thirteen (15\%) patients received surgery and systemic therapy at first recurrence, and six $(6 / 13,46 \%)$ of these developed a second recurrence. Nine (11\%) patients received RT and systemic therapy at first recurrence of which four $(4 / 9,44 \%)$ subsequently recurred. Three $(4 \%)$ received surgery, RT and systemic therapy combined; all $(3 / 3,100 \%)$ recurred again. Two (2\%) patients received best supportive care (BSC) only (Fig. 1).

Of the 13 patients who underwent surgery and systemic therapy at first recurrence, $8(8 / 13,62 \%)$ patients had surgery to no evidence of disease (NED) followed by adjuvant anti-PD-1 based therapy. Of these patients, only $25 \%(2 / 8)$ developed a second recurrence of melanoma.

Of the 29 patients that recurred locoregionally at first recurrence, $23(23 / 29,79 \%)$ patients underwent surgery to NED and 14 patients later developed distant disease (four locoregional and distant recurrence, 10 distant recurrence only).

Response to 1st line systemic therapy

Sixty-eight (80\%) patients received $\Pi$ or IT as $1^{\text {st }}$ line systemic therapy at either first or subsequent melanoma recurrence. Twenty-six (26/68, 38\%) patients received anti-PD-1 based therapy, including single agent anti-PD-1 or anti-PD-1 in combination with an investigational agent. Fourteen (14/68, $21 \%)$ patients received ipilimumab and nivolumab combination immunotherapy. Twelve $(12 / 68,18 \%)$ patients received ipilimumab monotherapy and $16(16 / 68,24 \%)$ patients received $\Pi$. Of the 16 patients who received $\Pi$ as 1 st line systemic therapy after recurrence, only $5(5 / 16,31 \%)$ received drug alone, one of whom had a response (1 partial response (PR), 1 stable disease (SD), 3 progressive disease (PD)). 


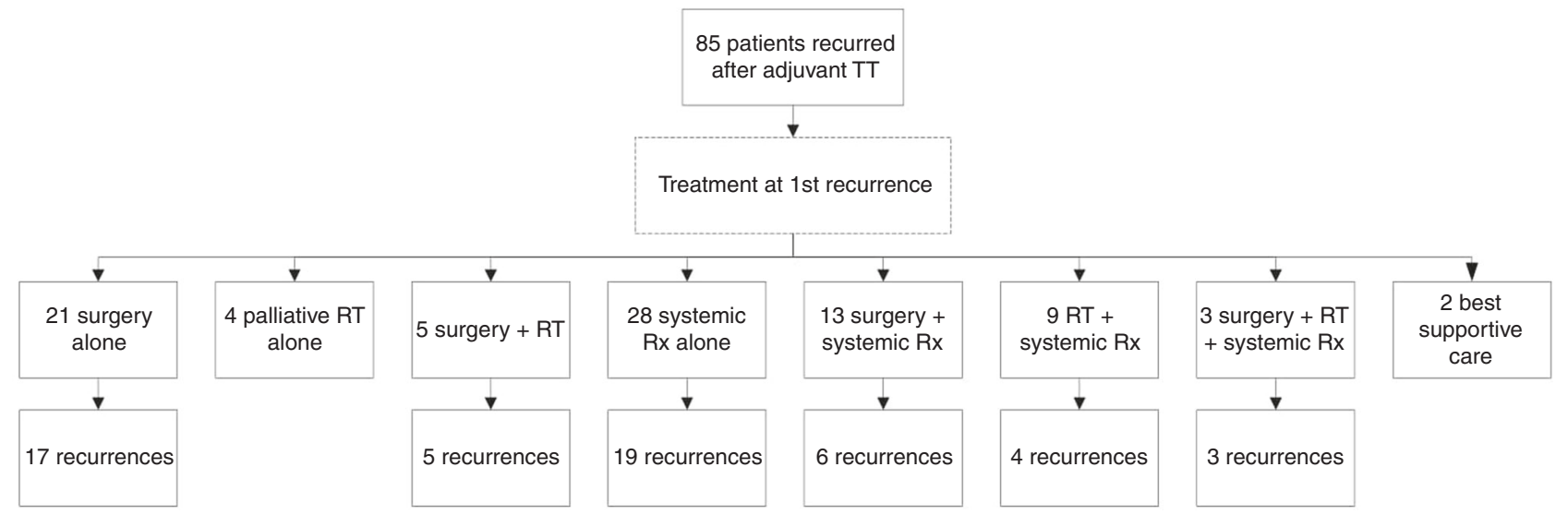

Fig. 1 Flow chart of treatment at first recurrence of melanoma after adjuvant TT. Rx therapy, RT radiotherapy, TT targeted therapy.

Response rate (RR) to 1 st line systemic therapy after relapse was deemed assessable if patients received $\Pi$ or IT at first or subsequent recurrence (with no prior systemic therapy) and had measurable disease for assessment. Two patients received adjuvant interferon at first recurrence followed by IT at second recurrence; these patients were also included in determining RR. Twenty-seven (32\%) patients were not assessable for response: two received BSC, three received RT only for recurrence, five received surgery only, one underwent surgery at first recurrence then RT at second recurrence, five received non-IT or $\Pi \mathrm{T}$ as $1 \mathrm{st}$ line systemic therapy (3 temozolomide, 1 interferon, 1 TVEC monotherapy), nine received surgery followed by adjuvant therapy (best response NED) and two patients completed treatment before disease assessment (both ipilimumab monotherapy).

Thus, $58(68 \%)$ patients received IT or $\Pi$ as 1 st line systemic therapy at either first or subsequent recurrence and had disease that was assessable for response. RR to anti-PD-1 therapy, either as monotherapy or in combination with a trial agent was $63 \%$, whilst RR to ipilimumab and nivolumab combination immunotherapy was $62 \%$. RR to a rechallenge of $\Pi$ was $25 \%$ and RR to single agent ipilimumab was $10 \%$ (Table 3 ). Of the 19 patients with evaluable disease who received anti-PD-1 based therapy, 6 (32\%) were on a clinical trial involving an anti-PD-1 agent in combination with an investigational agent. Of these six patients, one had a complete response (CR) and 5 had a partial response (PR)- thus, $100 \%(6 / 6)$ had a response to anti-PD-1 and trial agent therapy. Of the thirteen patients who received anti-PD-1 monotherapy as 1st line systemic therapy, RR was $46 \%(6 / 13)$. Median time to first recurrence from start of adjuvant $\Pi$ was 23 months for anti-PD-1 ( \pm trial) therapy, 18 months for ipilimumab and nivolumab combination therapy, 13 months for $\pi$ rechallenge and 13 months for ipilimumab monotherapy.

Of the 21 patients who underwent surgery alone at first recurrence with no immediate adjuvant therapy, 9 (9/21, 43\%) received subsequent anti-PD-1 based therapy as 1st line systemic therapy at second or subsequent relapse. Eight $(8 / 9,89 \%)$ of these patients responded to this subsequent anti-PD-1 therapy $(5 C R$, 3 PR).

Overall survival

Median overall survival (mOS) from the date of first recurrence for all patients was not reached. Two-year OS for all patients from date of first recurrence was $71 \%$ (Supplement 2). Twenty-eight (33\%) patients had died at census, all due to melanoma. Median follow-up from time of first recurrence was 31 months.

OS was not significantly different between patients who received single agent or combination $\Pi$ as adjuvant therapy $(p=0.25)$.
There was also no significant difference in OS between patients who relapsed locoregionally or distally at first recurrence after adjuvant $\Pi$ (Supplement 3; $p=0.16$ ).

OS varied by drug class received as 1 st line systemic therapy at relapse (Fig. 2). Two-year OS was $84 \%$ for anti-PD-1 therapy with or without a trial agent, $92 \%$ for ipilimumab and nivolumab combination immunotherapy, $49 \%$ for $\Pi$ and $45 \%$ for ipilimumab monotherapy $(p=0.028)$. This remained significant in multivariant analysis for $\operatorname{sex}(p=0.043)$.

Recurrence during vs after adjuvant $\Pi$

No statistically significant difference in mOS occurred between patients who had recurrent melanoma whilst still receiving adjuvant $\Pi$ compared to those who recurred after ceasing adjuvant $\Pi$ ( $p=0.20$, Fig. 3$)$.

\section{DISCUSSION}

The choice of adjuvant therapy for patients with BRAF mutant stage III melanoma is unclear, with compelling evidence supporting use of either $\Pi$ or IT. Both approaches have led to similar relative risk reductions in RFS, and thus the decision between TT and IT is often dependant on patient and clinician preference. Many clinicians favour IT due to the sustained improvements in PFS and OS seen in the metastatic setting. It is important to note, however, that data is not yet available on whether adjuvant antiPD-1 therapy leads to an improvement in OS. In contrast, COMBI$A D$ demonstrated an improvement in OS for adjuvant $\Pi T$ with a HR of $0.57(p=0.0006)$, though this did not reach the pre-specified significance boundary.

One factor of relevance when deciding between adjuvant $\pi$ and IT for BRAF mutant patients is knowledge of RRs and survival rates to subsequent systemic therapy upon relapse after adjuvant therapy. Owen et al presented retrospective data on patients who relapsed after adjuvant anti-PD-1 therapy, demonstrating that no patients responded to subsequent single agent anti-PD-1 if relapse occurred whilst on adjuvant anti-PD-1, whereas $40 \%$ of patients responded to a re-challenge of anti-PD-1 therapy if relapse occurred after cessation of adjuvant anti-PD-1. Interestingly, $79 \%$ of patients responded to $\Pi$ if relapse occurred on adjuvant anti-PD-1, whilst $88 \%$ responded to $\Pi$ after ceasing antiPD-1 therapy. ${ }^{18}$ Thus, a change in treatment modality to either combination IT or $\Pi$ is generally recommended in those patients that relapse on or after adjuvant single agent IT.

To our knowledge, this is the first study to report the RRs of BRAF mutant melanoma patients to subsequent systemic therapy following relapse after adjuvant $T$. The RR of patients to anti-PD-1 based immunotherapy (anti-PD-1 with or without trial agent) after 
Table 3. Response rates to 1 st line systemic therapy after melanoma recurrence.

\begin{tabular}{lcccc}
\hline & $\begin{array}{l}\text { Anti-PD-1 } \pm \text { trial drug } \\
(N=19)\end{array}$ & $\begin{array}{l}\text { Ipilimumab + Nivolumab } \\
(N=13)\end{array}$ & $\begin{array}{l}\text { Targeted therapy } \\
(N=16)\end{array}$ & $\begin{array}{l}\text { Ipilimumab single agent } \\
(N=10)\end{array}$ \\
\hline Complete Response (CR) & $4(21 \%)$ & $4(31 \%)$ & $1(6 \%)$ & $1(10 \%)$ \\
Partial Response (PR) & $8(42 \%)$ & $4(31 \%)$ & $3(19 \%)$ & 0 \\
Stable Disease (SD) & 0 & 0 & $1(6 \%)$ & $1(10 \%)$ \\
Progressive Disease (PD) & $7(37 \%)$ & $5(38 \%)$ & $11(69 \%)$ & $8(80 \%)$ \\
Response Rate (RR) & $63 \%(12 / 19)$ & $62 \%(8 / 13)$ & $25 \%(4 / 16)$ & $10 \%(1 / 10)$ \\
\hline
\end{tabular}

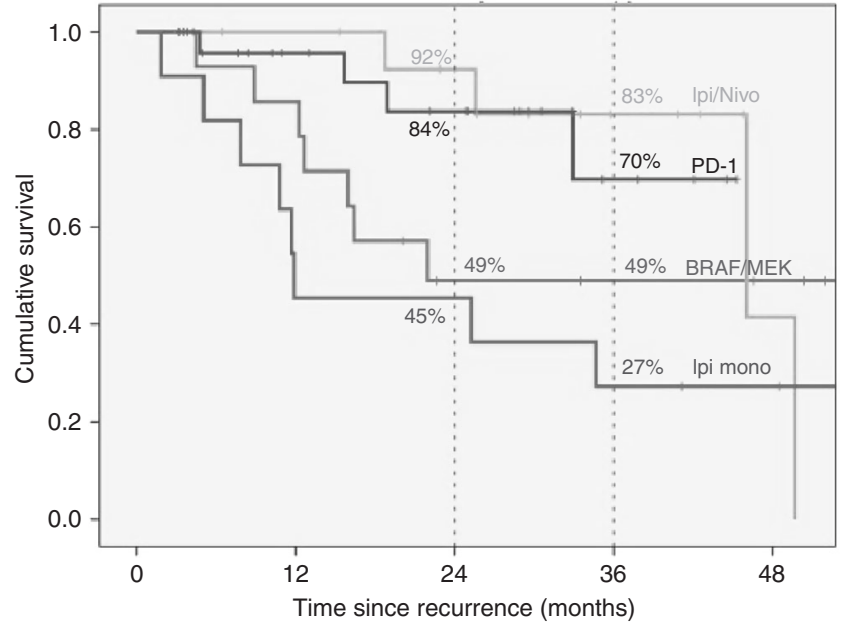

Fig. 2 Kaplan-Meier curve of overall survival for all patients from time of first melanoma recurrence, by class of $1^{\text {st }}$ line systemic therapy received at recurrence $(p=0.028)$.

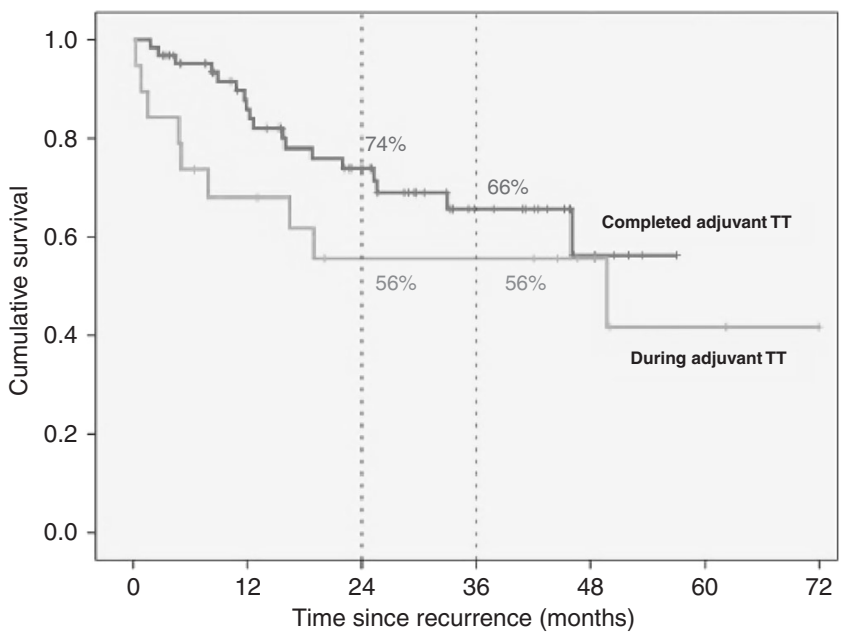

Fig. 3 Kaplan-Meier curve of overall survival for patients who developed melanoma recurrence during and after adjuvant TT ( $p=$ 0.20).

relapse was $63 \%$ and the RR to ipilimumab-nivolumab combination therapy was $62 \%$, demonstrating that patients treated with adjuvant TT remain sensitive to IT. All patients (20/20, 100\%) who responded to anti-PD-1 based IT as 1st line systemic treatment had developed recurrent melanoma after cessation of adjuvant $\Pi$. Of those patients who had completed adjuvant $\Pi$ at recurrence, RR was $60 \%(6 / 10)$ to anti-PD-1 monotherapy (without a trial agent), $100 \%$ (6/6) for anti-PD-1 therapy with a trial agent and $73 \%$ $(8 / 11)$ to ipilimumab-nivolumab combination treatment. These results compare favourably to RRs in the metastatic, treatment naive setting, with Checkmate-067 demonstrating objective RRs of $67 \%$ to ipilimumab-nivolumab combination and $37 \%$ to nivolumab monotherapy in BRAF mutant patients. ${ }^{2}$ Furthermore, patients treated with systemic therapy after relapse had a twoyear OS rate of $92 \%$ for ipilimumab-nivolumab combination therapy and $45 \%$ for ipilimumab monotherapy. These results are also favourable when compared to those in Checkmate-067, where 2-year OS was $63 \%$ for ipilimumab- nivolumab and $43 \%$ for ipilimumab monotherapy. ${ }^{2}$

Of note, our study included two patients with resected stage IV melanoma, which varies from the cohort of patients included in most adjuvant trials. The COMBI-AD, EORTC 18071 and Keynote054 trials included patients with resected stage IIIA-C disease, with resected stage IV patients being excluded. Checkmate-238 is the only key adjuvant trial to have included resected stage IV patients, though this trial involved adjuvant IT not T. Despite our study including two patients with resected stage IV disease, these patients would not have influenced overall results as neither was assessable for response to 1st line systemic therapy at recurrenceone patient underwent resection of metastatic disease followed by adjuvant $\Pi$ (best response NED) and the second patient received TVEC monotherapy.

In patients with metastatic melanoma, response to 2 nd line single agent anti-PD-1 after 1 st line palliative $\pi$ is $\sim 25 \% .{ }^{19}$ This is significantly less than the RR of $37 \%$ seen in the 1 st line setting in BRAF mutant patients. This reduced efficacy after prior $\Pi$ is why some clinicians advocate for IT to be given upfront in the metastatic setting, and this reasoning is often extrapolated to the adjuvant space. However, our study suggests that the RR to IT after prior adjuvant $\Pi T$ is not diminished when compared to $1 \mathrm{st}$ line IT in the metastatic setting. Most patients who are treated with $\Pi$ in the metastatic setting are treated continuously until disease progression and are therefore truly resistant to BRAF/MEK therapy. In contrast, patients treated with adjuvant $\Pi$ infrequently relapse during treatment, with most relapses occurring after completion of therapy. Interestingly, in our study, no patients who developed recurrence whilst receiving adjuvant $\Pi$ responded to subsequent anti-PD-1 ( \pm trial agent) or ipilimumab-nivolumab combination therapy, though only five patients met this criterion (three patients had PD to anti-PD- $1 \pm$ trial; two patients had PD to ipilimumab-nivolumab; no patients had SD to either agent). This lends support to the notion that at relapse, the biology and immunogenicity of disease that recurs after completion of $\Pi T$ may be different to disease that progresses during $\pi^{20-24}$

In our cohort, $21 \%$ of patients ceased adjuvant $\Pi$ due to toxicity, similar to the $26 \%$ discontinuation rate due to AEs seen in COMBI-AD, noting that our cohort also included single agent $\Pi{ }^{16}$ Also, 22\% developed recurrent melanoma while receiving adjuvant $\Pi$. Of note, in our study, patients were specifically included only if they had developed recurrent melanoma. Interestingly, no statistically significant difference in OS occurred between patients relapsing during adjuvant $\Pi T$ or after completion of adjuvant $\Pi$ ( $p=0.20$, Fig. 3 ).

Patients who received surgery alone without systemic therapy after $1^{\text {st }}$ recurrence had a high relapse rate of $85 \%$. This suggests 
that surgery alone in the setting of melanoma that has relapsed after adjuvant $\Pi$ is unlikely to result in long term disease control, and a low threshold to initiate "adjuvant" systemic therapy at this time, or at least careful surveillance, is needed.

We found that the RR to subsequent $\Pi$, given as a rechallenge after relapse following adjuvant $\Pi$, was $25 \%$ (4/16). This is slightly less than the RR seen in other studies examining the efficacy of a $\Pi$ rechallenge in the stage IV setting; for example, Schreuer et al. ${ }^{25}$ reported a RR of $32 \%$ whilst Valpione et al. ${ }^{26}$ reported a RR of $43 \%$. The RR of $25 \%$ to a $\Pi$ rechallenge in our cohort is also lower than the RR of $68 \%$ to $\Pi$ seen in the $1^{\text {st }}$ line metastatic setting. ${ }^{5}$ These results suggest that patients who relapse after adjuvant $\Pi$ may benefit from a change in treatment modality rather than a rechallenge of $\Pi$.

Of the four patients who responded to a rechallenge with $\Pi$ at first recurrence, 75\% (3/4) had developed recurrent melanoma after ceasing adjuvant $\Pi$. All four patients had received combination $\Pi$ as adjuvant therapy. One patient relapsed whilst receiving adjuvant $\Pi$ and subsequently had a complete response to a rechallenge of $\Pi$ as 1 st line systemic therapy after relapse. However, between relapse and further $\Pi$, the patient underwent three surgical resections and adjuvant RT to locally recurrent melanoma. Thus, the interval between cessation of adjuvant $\Pi T$ and rechallenge with $\Pi$ was two years. These results suggest that maximising the time interval between adjuvant $\Pi$ and a rechallenge at recurrence may increase the likelihood of subsequent response.

The median time to recurrence from commencement of adjuvant systemic therapy in this cohort was 17.7 months. In patients who relapse after adjuvant anti-PD-1 therapy, median time to recurrence has recently shown to be much shorter, at 4.6 months. $^{18}$ This reflects the fact that recurrences on $\Pi$ are infrequent, with most recurrences occurring after adjuvant $\Pi$ is completed. In contrast, recurrences during adjuvant anti-PD-1 therapy are relatively more frequent than recurrence during adjuvant $\Pi$, with fewer recurrences occurring after cessation of adjuvant anti-PD-1. For example, Checkmate-238 demonstrated an RFS rate of $70 \%$ at the 1-year mark, compared to an RFS rate of $88 \%$ at 1 year in the COMBI-AD trial. ${ }^{13,17}$

Limitations of our study include the retrospective nature of data collection, and small sample size. Despite including 21 international sites, we were only able to recruit a modest number of patients, though this is expected given the relative infancy of adjuvant therapy in melanoma and the necessity to include BRAF mutant patients only. Imaging follow-up was performed approximately every 3 months, though not stringently regulated, at each patient's treatment centre and varied between CT, PET and MRI. Furthermore, measurement of response to subsequent therapy was performed by a patient's overseeing clinician, rather than a centralised review. Thus, heterogeneity in timing of tumour assessment and response evaluation would have occurred. The exception to this is patients enrolled on a clinical trial, where imaging frequency was stipulated in the respective trial protocol and response assessment occurred via usual Response Evaluation Criteria in Solid Tumours (RECIST) measurements.

\section{CONCLUSIONS}

This study suggests that patients who relapse after adjuvant $\Pi T$ respond to subsequent IT-based therapy at comparable rates to the 1st line or treatment naïve setting. Therefore, when considering choice of adjuvant systemic therapy in BRAF mutant melanoma patients, clinicians should consider TT as an option that may not diminish the chance of response to subsequent IT. Furthermore, switching to PD-1 based IT after relapse results in superior RRs and survival than further $\Pi$ or ipilimumab monotherapy. More evidence is needed to clarify the optimal approach to managing patients with recurrent melanoma after adjuvant $\Pi$. Further data on this topic is likely to become available as adjuvant therapy is increasingly utilised and longer follow-up of randomised trials investigating adjuvant $\Pi$ occurs. Ultimately, decisions about adjuvant therapy for resected stage III/IV BRAF mutant melanoma patients should be made on an individual basis, wherein the choice between adjuvant IT and $\Pi$ takes into consideration potential toxicities, costs and patient preference. ${ }^{27}$

\section{ACKNOWLEDGEMENTS}

An abstract of this study was presented at a poster discussion session during the American Society of Clinical Oncology Annual Scientific Meeting 2020.

\section{AUTHOR CONTRIBUTIONS}

P.B. conceived the project; collected, collated and analysed the data, wrote and edited the original paper. L.P. provided data and edited the paper. G.V.L. revised the paper and helped in result interpretation. A.M.M. revised the paper and helped in result interpretation. V.A. provided data and edited the paper. J.V.C. provided data and edited the paper. R.J.S. revised the paper and helped in result interpretation. V.CS. provided data and edited the paper. MN provided data and edited the paper. K.K. provided data and edited the paper. A.H. revised the paper and helped in result interpretation. R.P. provided data and edited the paper. C.T. provided data and edited the paper. P.A.A. revised the paper and helped in result interpretation. L.Z. provided data and edited the paper. D.S. revised the paper and helped in result interpretation. C.A. provided data and edited the paper. C.L. revised the paper and helped in result interpretation. A.M. provided data and edited the paper. M.S. revised the paper and helped in result interpretation. S.R. provided data and edited the paper. C.R. revised the paper and helped in result interpretation. T.L. provided data and edited the paper. S.P. provided data and edited the paper. J.M.V. provided data and edited the paper. C.U.B. revised the paper and helped in result interpretation. A.K. provided data and edited the manuscript. A.V.W. provided data and edited the paper. M.S.C. provided data and edited the paper. M.S. revised the paper and helped in result interpretation. A.H. conceived the project, played an important role in data analysis, wrote and edited the original paper. All authors approved the final version of the paper and agreed to be accountable for all aspects of the work, including ensuring that questions related to the accuracy or integrity of any part of the work are appropriately investigated and resolved.

\section{ADDITIONAL INFORMATION}

Ethics approval and consent to participate Ethics board approval was obtained for this project from Alfred Health. The requirement for consent from subjects to participate in the project was waived by the ethics board. The study was performed in accordance with the Declaration of Helsinki.

Data availability Data supporting the results reported in this article is stored at Alfred Health and can be provided upon request.

Competing interests P.B.: Travel support: MSD G.V.L.: Consultancy: Aduro, Amgen, Bristol-Myers Squibb, Mass-Array, Merck, MSD, Novartis, OncoSec Medical, Pierre Fabre, Roche, QBiotics, Skyline DX and Sandoz. A.M.M.: Consultancy: BMS, MSD, Novartis, Roche, Pierre-Fabre. Acknowledgement Cancer Institute NSW Fellowship and Melanoma Institute Australia. VA. Consultancy: BMS, MSD, Merck, Novartis, Pierre Fabre, Roche, NEKTAR. Speaker honoraria: BMS, MSD, Merck, Novartis. Travel support: BMS, Oncosec. J.V.C.: Consultancy: Sanofi-Genzyme, BMS. R.J.S.: Consultancy: Asana Biosciences, Bristol Myers Squibb, Novartis, Merck, Lovance. Research funding: Merck, Amgen. M.N.: Consultancy: Novartis, BMS, Pierre-Fabre. Speaker honoraria: Novartis, BMS, Pierre-Fabre. K.K.: Personal fees: Amgen, Roche, Bristol Myers Squibb, Merck Sharp and Dohme, Novartis, Amgen, Pierre Fabre, Medac. A.H.: Consultancy: Amgen, BMS, MerckSerono, MSD/Merck, Philogen, Pierre Fabre, Provectus, Regeneron, Roche, OncoSec, Sanofi-Genzyme, Sun Pharma, Novartis Pharma, Almirall Hermal. Speaker honoraria: Amgen, BMS, MSD/Merck, Pierre Fabre, Provectus, Regeneron, Roche, Sanofi-Genzyme Novartis Pharma. Research funding: Amgen, BMS, MerckSerono, MSD/Merck, Philogen, Pierre Fabre, Provectus, Regeneron Roche, Sanofi-Genzyme, Novartis Pharma. R.P.: Consultancy: Pierre Faber, Bayer, Octimet, Clovis Oncology, Novartis, Karus Therapeutics, Biosceptre, BMS, Cybrexa, Ellipses, CV6 Therapeutics, Astex Therapeutics, Sanofi Aventis. Speaker honoraria: AstraZeneca, Novartis, Bayer, Tesaro, BMS. Travel support: BMS, MSD. P.A.A.: Consultancy: Bristol Myers-Squibb, Roche-Genentech, Merck Sharp \& Dohme, Array, Novartis, Merck Serono, Pierre Fabre, Incyte, NewLink Genetics, Genmab, Medimmune, AstraZeneca, Syndax, SunPharma, Sanofi, Idera Ultimovacs, Sandoz, Immunocore, 4SC, Alkermes, Italfarmaco, Nektar, Boehringer- 
Ingelheim. Research funding: Bristol Myers-Squibb, Roche-Genentech, Array. Travel support: MSD. L.Z.: Consultancy: BMS, Novartis, Pierre Fabre, Sunpharma, Sanofi, MSD. Honoraria from Roche, BMS, MSD, Novartis, Pierre Fabre. Research funding: Novartis; Travel support: BMS, Pierre Fabre, Sanofi, Amgen, Novartis, Sunpharma. D.S.: Consultancy: BMS, Merck Serono, Amgen, Immunocore, Incyte, 4SC, Pierre Fabre, Mologen, Merck/MSD, Sanofi/Regeneron. Speaker honoraria: Roche/ Genentech, Novartis, BMS, Merck Serono, Amgen, Immunocore, Incyte, 4SC, Pierre Fabre, Array BioPharma, InFlarX, Philogen, Regeneron, Merck/MSD, Sandoz/Hexal, Neracare. Research funding: Novartis, BMS. Travel support: Roche/Genentech, Novartis, BMS, Merck Serono, Amgen, Merck/MSD. C.A.: Travel support: Amgen, BMS, Roche. C.L.: Consultancy: Avantis Medical Systems, BMS, MSD, Novartis, Amgen, Roche, Merck Serono, Sanofi. Research funding: BMS, Roche. Speaker honoraria: BMS, MSD, Novartis, Amgen, Roche, Pierre Fabre, Pfizer, Incyte. Travel support: BMS, MSD, CR: Consultancy: BMS, Pierre Fabre, Novartis, Merck, MSD, Roche, Sanofi, Biothera, Curevac. T.L.: Consultancy: MSD, Pierre Fabre, Novartis. S.P.: Speaker honoraria: Merck CUB: Consultancy: BMS, MSD, Roche, Novartis, GSK, AZ, Pfizer, Lilly, GenMab, Pierre Fabre, Third Rock Ventures. Research funding: BMS, Novartis, NanoString. Stockowner: Uniti Cars. A.K.: Speaker honoraria: Novartis. Travel support: Novartis. A.V.W.: Travel support: BMS, Novartis, MSD. M.S.C.: Consultancy: BMS, MSD, Novartis, Amgen, Roche, Pierre Fabre, Nektar, Eisia, Sanofi, Merck Serono, Ideay, Q biotics. M.S.: Consultancy: BMS, Merch, MSD. Speaker honoraria: BMS, Merck, MSD. Research funding: BMS. Travel support: BMS, Merck, Roche. A.H.: Consultancy: Novartis, BMS, MSD, Pierre-Fabre. All remaining authors have declared no conflict of interest.

Funding information G.V.L.: acknowledgements: NHMRC Practitioner Fellowship, the University of Sydney Medical Foundation and Melanoma Institute Australia. A.M.M.: Acknowledgements: Cancer Institute NSW Fellowship and Melanoma Institute Australia.

Supplementary information is available for this paper at https://doi.org/10.1038/ s41416-020-01121-y.

Note This work is published under the standard license to publish agreement. After 12 months the work will become freely available and the license terms will switch to a Creative Commons Attribution 4.0 International (CC BY 4.0).

Publisher's note Springer Nature remains neutral with regard to jurisdictional claims in published maps and institutional affiliations.

\section{REFERENCES}

1. Wolchok, J. D., Chiarion-Sileni, V., Gonzalez, R., Rutkowski, P., Grob, J.-J., Lance Cowey, C. et al. Overall survival with combined nivolumab and ipilimumab in advanced melanoma. N. Engl. J. Med. 377, 1345-1356 (2017).

2. Larkin, J. M. G., Chiarion-Sileni, V., Gonzalez, R., Grob, J.-J., Rutkowski, P., Lao, C. D. et al. 5-year survival outcomes of the CheckMate 067 phase 3 trial of nivolumab plus ipilimumab (NIVO+IPI) combination therapy in advanced melanoma. N. Engl. J. Med. 381, 1535-1546 (2019).

3. Long, G. V., Flaherty, K. T., Stroyakovskiy, D., Gogas, H., Levchenko, E., de Braud, F. et al. Dabrafenib plus trametinib versus dabrafenib monotherapy in patients with metastatic BRAF V600E/K-mutant melanoma: long-term survival and safety analysis of a phase 3 study. Ann. Oncol. 28, 1631-1639 (2017).

4. Grob, J. J., Amonkar, M. M., Karaszewska, B., Schachter, J., Drummer, R., Mackiewicz, A. et al. Comparison of dabrafenib and trametinib combination therapy with vemurafenib monotherapy on health-related quality of life in patients with unresectable or metastatic cutaneous BRAF Val600-mutation-positive melanoma (COMBI-v): results of a phase 3, open-label, randomised trial. Lancet Oncol. 16 , 1389-1398 (2015).

5. Robert, C., Grob, J. J., Stroyakovskiy, D., Karazewska, B., Hauschild, A., Levchenko, E. et al. Five-year outcomes with dabrafenib plus trametinib in metastatic melanoma. N. Engl. J. Med. 381, 626-636 (2019).

6. Gershenwald, J. E., Scolyer, R. A., Hess, K. R., Sondak, V. K., Long, G. V., Ross, M. I. et al. Melanoma staging: evidence-based changes in the american joint committee on cancer eighth edition cancer staging manual. CA Cancer J. Clin. 67, 472-492 (2017).

7. Kanaki, T., Stang, A., Gutzmer, R., Zimmer, L., Chorti, E., Sucker, A. et al. Impact of American joint committee on cancer 8th edition classification on staging and survival of patients with melanoma. Eur. J. Cancer 119, 18-29 (2019).
8. Eggermont, A. M. M., Chiarion-Sileni, V., Grob, J. J., Drummer, R., Wolchok, J. D., Schmidt, $\mathrm{H}$. et al. Adjuvant ipilimumab versus placebo after complete resection of high-risk Stage III melanoma (EORTC 18071): a randomised, double-blind, phase 3 trial. Lancet Oncol. 16, 522-530 (2015).

9. Eggermont, A. M. M., Chiarion-Sileni, V., Grob, J. J., Drummer, R., Wolchok, J. D., Schmidt, $\mathrm{H}$. et al. Prolonged survival in stage III melanoma with ipilimumab adjuvant therapy. N. Engl. J. Med. 375, 1845-1855 (2016).

10. Eggermont A. M. M., Chiarion-Sileni V., Grob, J. J., Drummer R., Wolchok J. D., Schmidt $\mathrm{H}$. et al. Ipilimumab versus placebo after complete resection of stage III melanoma: long-term follow-up results the EORTC 18071 double-blind phase 3 randomized trial. J. Clin. Oncol. 37, abstract 2512 (2019)

11. Tarhini, A. A., Lee, S. J., Hodi, F. S., Rao, U. N. M., Cohen, G. I., Hamid, O. et al. Phase III study of adjuvant ipilimumab ( 3 or $10 \mathrm{mg} / \mathrm{kg}$ ) versus high-dose interferon alfa$2 \mathrm{~b}$ for resected high-risk melanoma: North American Intergroup E1609. J. Clin. Oncol. 38, 567-575 (2019).

12. Weber, J., Mandala, M., Del Vecchio, M., Gogas, H. J., Arance, A. M., Cowey, C. L. et al. Adjuvant nivolumab versus ipilimumab in resected stage III or IV melanoma. N. Engl. J. Med. 377, 1824-1835 (2017).

13. Weber, J., Del Vecchio, M., Mandala, M., Gogas, H., Arance, A. M., Dalle, S. et al. Adjuvant nivolumab (NIVO) versus ipilimumab (IPI) in resected stage III/IV melanoma: 3-year efficacy and biomarker results from the phase III CheckMate 238 trial. Ann. Oncol. 30(Suppl. 5), v533-563 (2019).

14. Eggermont, A. M. M., Blank, C. U., Mandala, M., Long, G. V., Atkinson, V., Dalle, S. et al. Adjuvant pembrolizumab versus placebo in resected stage III melanoma. $N$. Engl. J. Med. 378, 1789-1801 (2018).

15. Maio, M., Lewis, K., Dernidov, L., Mandala, M., Bondarenko, I., Ascierto, P. A. et al. Adjuvant vemurafenib in resected, BRAFV600 mutation-positive melanoma (BRIM8): a randomised, double-blind, placebo-controlled, multicentre, phase 3 trial. Lancet Oncol. 19, 510-520 (2018).

16. Long, G. V., Hauschild, A., Santinami, M., Atkinson, V., Mandala, M., Chiarion-Sileni, V. et al. Adjuvant Dabrafenib plus trametinib in stage III BRAF-mutated melanoma. N. Engl. J. Med. 377, 1813-1823 (2017).

17. Hauschild, A., Drummer, R., Schadendorf, D., Santinami, M., Atkinson, V., Mandala, $M$. et al. Longer follow-up confirms relapse-free survival benefit with adjuvant dabrafenib plus trametinib in patients with resected BRAF V600-mutant stage III melanoma. J. Clin. Oncol. 36, 3441-3449 (2019).

18. Owen C. N., Shoushtri A. N., Chauhan D., Palmieri D. J., Lee B., Rohaan M. W. et al. Management of early melanoma recurrence despite adjuvant anti-PD-1 antibody therapy. Ann. Oncol. https://doi.org/10.1016/j.annonc.2020.04.471 (2020)

19. Hamid, O., Robert, C., Daud, A., Hodi, F. S., Hwu, W. J., Kefford, R. et al. Five-year survival outcomes for patients with advanced melanoma treated with pembrolizumab in KEYNOTE-001. Ann. Oncol. 30, 582-588 (2019).

20. Frederick, D. T., Piris, A., Cogdill, A. P., Cooper, Z. A., Lezcano, C., Ferrone, C. R. et al. BRAF inhibition is associated with enhanced melanoma antigen expression and a more favorable tumor microenvironment in patients with metastatic melanoma. Clin. Cancer Res. 19, 1225-1231 (2013).

21. Wilmott, J. S., Long, G. V., Howle, J. R., Haydu, L. E., Sharma, R. N., Thompson, J. F. et al. Selective BRAF inhibitors induce marked T-cell infiltration into human metastatic melanoma. Clin. Cancer Res. 18, 1386-1394 (2012).

22. Kakavand, H., Rawson, R. V., Pupo, G. M., Yang, J. Y. H., Menzies, A. M., Carlino, M. S. et al. PD-L1 expression and immune escape in melanoma resistance to MAPK inhibitors. Clin. Cancer Res. 23, 6054-6061 (2017).

23. Hugo, W., Shi, H., Sun, L., Piva, M., Song, C. Y., Kong, X. et al. Non-genomic and immune evolution of melanoma acquiring MAPKi resistance. Cell 162, 1271-1285 (2015).

24. Shi, H., Hugo, W., Kong, X., Hong, A., Koya, R. C., Moriceau, G. et al. Acquired resistance and clonal evolution in melanoma during BRAF inhibitor therapy. Cancer Discov. 4, 81-93 (2015).

25. Schreuer, M., Jansen, Y., Planken, S., Chevolet, I., Seremet, T., Kruse, V. et al. Combination of dabrafenib plus trametinib for BRAF and MEK inhibitor pretreated patients with advanced $\mathrm{BRAF}^{\mathrm{V} 600}$-mutant melanoma: an open-label, single arm, dual-centre, phase 2 clinical trial. Lancet Oncol. 18, 464-472 (2017).

26. Valpione, S., Carlino, M. S., Mangana, J., Mooradian, M. J., McArthur, G., Schadendorf, D. et al. Rechallenge with BRAF-directed treatment in metastatic melanoma: A multi-institutional retrospective study. Eur. J. Cancer 91, 116-124 (2018).

27. Bhave, P., Pallan, L., Atkinson, V., Cohen, J. V., Chiarion-Sileni, V., Nyakas, M. et al. Melanoma recurrence after adjuvant targeted therapy: a multicenter analysis. J. Clin. Oncol. 38, abstract 10016 (2020). 